\title{
Toward A Fulldmplementation of Location Enabled Service Framework in 1 IMS Infrastructure
}

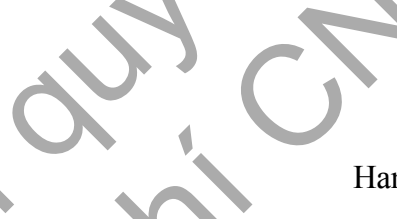

\author{
Nguyen Tai Hung \\ Hanoi University of Science and Technology, Hanoi, Vietnam \\ hung.nguyentai@hust.edu.vn
}

Abstract - IMS has been widely recognized as the control and brokering framework for delivering of the blended communication and multimedia services to the fix and mobile IP users. However, until recently, most of IMS services have been developed and deployed in a manner that does not carefully consider how these services can be combined and interact with each other in a beneficial way. Services can often overlap in terms of the logic that they execute, causing of redundant code across several application servers. In reference to OMA recommendation, we developed a hybrid approach to divide services into two categories of user services and enabling services. In that regard and in scope of our national research project, we had proposed and prototyped several service enablers for a composited and intelligent service platform using IMS infrastructure. In this paper, we are detailing of the proposed design of a Location Enabler and its interaction with other functional blocks of IMS framework to provide the advanced location enabled services to end users. The so-called Location Enabler collects data about the geographical position of mobile terminals and provided this information to requesting services/clients, via application server platform. Since location data can be regarded as a type of presence information, we propose an architecture that builds on the IMS presence specifications. We show that several mechanisms for processing presence like notification handling, access control and privacy management apply to location data as well. It is therefore possible to reuse a large part of the IMS presence infrastructure.

Keywords - IMS; LBS; Service Enabler; Location Enabled Services; Combined Services; Service Capabilities

\section{INTRODUCTION}

Traditionally, Location Based Services (LBS) provide added value mainly by using the physical position of mobile users. Location data may consist of plain geographical coordinates, access point, cell IDs, civil location in form of postal addresses or status like 'in the office', 'at home'. Examples of services are a map showing the user's current location or changing the handling of incoming calls when the user enters a specified area. Service enablers, defined to expose network functionality to external service providers, are becoming the cornerstones of modern service architectures defined by the Parlay Group [22], the Open Mobile Alliance (OMA) [6] or in the IP Multimedia Subsystem (IMS). A Location Enabler is a functional entity in the network enabling valueadded services to query the current position of a user or to request a trigger when a specified area is entered or left. Current standardization in the 3rd Generation Partnership Project (3GPP) [8] concentrates on Location Service Enablers that use core network components to provide location data of a user's terminal. For advanced notification-based location processing, we show that our distributed, terminalbased architecture scales better, is more accurate, efficient and cost effective. Location information provides considerable value to information and communication services. On the other hand, users are concerned about revealing their position data to others, especially to un-trusted third party applications. Furthermore, most countries have legal restrictions that regulate processing of personal data and the protection of privacy in electronic communications. Thus, it is of very importance that the users can control who gets access to their location data and that the transport in the network of such sensitive data is protected by strong security mechanisms.

The target of our service architecture is based on the IP Multimedia Subsystem (IMS) [1][4], an overlay network on top of the UMTS packet service (GPRS/3G/LTE). It has been specified by the 3GPP 
in the past years and is currently in the deployment phase. The IMS makes heavy use of the Session Initiation Protocol (SIP) [2] and its extensions and defines several service enablers such as presence, instant messaging, push to talk, etc. Whereas most UMTS applications can be realized without the IMS, the specified mechanisms promise a uniform, standardized way of handling quality of service, charging, roaming and integration of different services.

Our analysis of the IMS presence system shows that the requirements regarding access authorization, encryption and privacy for presence are indeed identical to those for location. The concept of presence was introduced in instant messaging systems. Presence is information about the online status of other users. A watcher (user) can subscribe to notifications about the state of another user. The watched user is called 'presentity'. Classical presence information is defined as the willingness of the presentity to communicate.

This paper proposes a comprehensive and reference framework of a service platform that using IMS technology and more importantly including the design and implementation of the Location Enablers and its integrated charging and policy control mechanisms. Our proposal has following advantages in regard of location enabled services compare to other approaches:

- Using distributed and terminal-based location data collection

- Using service (capabilities) composition mechanisms to provide more advanced \& orchestrated services to end user

- First ever proposed unified-architecture which combines location enabling concepts (similar to OMA) with charging and policy control mechanisms to make sure the services are deliverable and controllable

The paper is divided into five main sections. In Section II we list the major previous works on the topic, Section III summarizes the most important things about service architecture as well as its related interfaces/reference points in overall IMS framework and OMA, Section IV presents our design of a comprehensive architecture that combine advanced concepts of service providing, service enabling, service blending, service charging and policy control. Section $\mathrm{V}$ give the details of conceptual design and implementation of the location enabler and related the location enabled applications. Finally, Section VI describes our unified and federated Test-bed environment as well as some major prototyping results.

\section{PREVIOUS WORK}

Telecom operators are currently trying to build business models around new Web 2.0 and social network technologies. Banerjee et al [11] discuss a possible architecture involving telecom mash-ups. However, it does not based on IMS as the service framework to take advantage of the associated benefits. Shin et al. [10] describe the end-user driven service creation method for converged service over telecom and Internet domain. It focuses on service layer and takes advantage of the open network architecture but does not utilize IMS's rich call control feature. Our work, though not deal directly with Web 2.0 signaling and service control mechanisms, but takes advantage of the flexibility and openness of Parlay-X API and blends it with the SIP call control features of IMS. Polk and Rosen present a framework and requirements for usage of SIP to convey user location information and consider cases where message routing by intermediaries is influenced by the location of the session initiator [17].

Surprisingly, to our best knowledge, there are not many research works on this IMS-based Location Enabled Services (LBS) recently, most of the updated researches was published in 2009-2010. The reason may be the fact that LBS is already well established technology so most of activities now are focusing on how to deploy and to make profit from it. However, on our view, this is just the beginning for LBS; the increasing sophistication of the smart phone, highspeed data rates with LTE and consumer demand for 'always-on' interaction mean that LBS applications are going to expand and optimize massively over the coming years. Furthermore, in our opinion, in order for a successful commercial deployment (of LBS) in reality, there is a need to complete the service architecture with essential functions of charging and policy control and our work is just for those aims.

One of the other work field that is important to the location enabled services are the service invocation and blending mechanisms that allows system to combine multiple sessions between Web 2.0/Parlay-X and communication platform to provide 


\section{Research, Development and Application on Information and Communications Technology}

end user with services like Mash-up. On our research we use the IMS as service control infrastructure to utilize its strength on this type of job. The IMS currently only supports static chaining of services using initial Filter Criteria (iFC) techniques [13]. The static service chaining technique relies on the SCSCF to take on the burden of monitoring all executing services. In this model, Application Servers (AS's) are not aware of each other and may only communicate via the S-CSCF. There is therefore limited capacity for cooperative execution of services via service blending and/or composition. In order to address this problem the 3GPP has recently introduced a new functional entity to the IMS called the Service Capability Interaction Manager or SCIM [14]. The SCIM is envisioned as a network function that will allow dynamic blending of services and therefore support some form of service composition. However, the 3GPP has only provided a high level definition of the functionality of the SCIM until the time of this writing. The need for a detailed specification of the SCIM has therefore produced a variety of research describing SCIM-based brokering architectures. Some researchers have proposed Service Brokering as a functional element of the SCIM tasked with creating a workflow for blended services [15]. However the latter approach creates a bottleneck in the network due to its centralized architecture. A more distributed approach would alleviate the burden placed on the SCIM that results in low fault tolerance when orchestrating blended services [16]. In fact increasingly sophisticated systems have been proposed that rely on distributed solutions to the problem of Service Brokering [17]. The blended service SCIM approach limits the Service Broker to coordinating the interaction of features amongst service capabilities as opposed to specifically creating new services based on service requests, which is the essence of Service Brokering [18]. Our approach [23] for this problem is to make use of OMA-compliant service enablers in combination with IMS service invocation mechanisms in order to provide a scalable and expandable architecture in terms of more advanced blending rules as well as more rapid service development process.

The remainder of this paper describes basic concepts of IMS based service architecture especially the service enablers. We then describe our design and implementation of Location Enabler and the illustrated typical location enabled services. Finally, our live test-bed and prototyped results are provided, follows by conclusion and lessons learned.

\section{IMS SERVICES ARCHITECTURE}

\section{A. Services Platform and Interfaces}

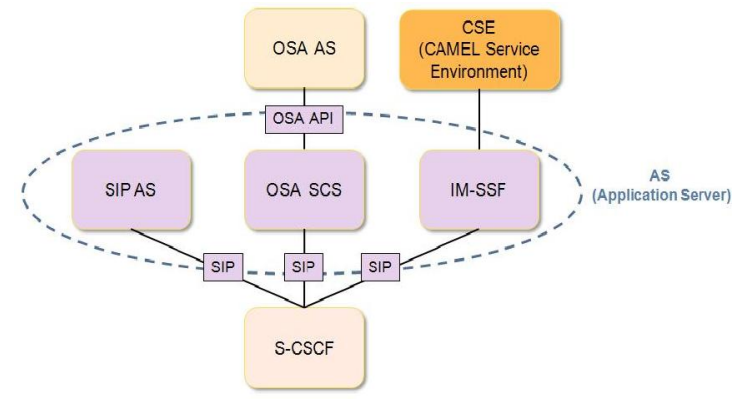

Figure 1. IMS Service Delivery Framework

The IMS service plane [1] basically contains all application servers and gateways used for service execution. As explained in many available documents, the S-CSCF is the call server from the control plane responsible for interfacing with the service plane across the ISC interface (SIP protocol). Although service plane is accessed by making use of the SIP protocol, service gateways allow having application servers that are not necessary SIP-native. These gateways may be of different types and facilitate the reuse of legacy service platforms and/or Internet.

Beside these gateways, IMS also defined an additional functional element:

- $\quad$ SCIM (Service Capability Interaction Manager): This optional element manages the interaction between the different Application Servers more efficiently than the S-CSCF can do.

Among the others, the OSA SCS is the important gateway to allow the services hosted on the Parlay and ParlayX platform to be executed and delivered to mobile IMS users. This gateway is crucial because it supports the connectivity between the limited Web 2.0 (via ParlayX API) environment to the IMS (telecom) framework and this leads to the popularity of the so called Mash-up services/apps currently. Mash-up services allow Web 2.0 users to initiate telecom services (phone call, messaging, video sharing, etc) during and on the session of web surfing. 
In other word, a "telecom mash-up" is a telecommunications service where service elements come from more than one source and are combined an integrated experience. For example, one could get the base service from company $A$, a ring-back tone from company B, a voicemail service from company $\mathrm{C}$, etc. It is now widely thought that the deployment of IMS can benefit greatly from the blending of its rich call control features with the existing Web 2.0 or "mash up" applications that are based on open API. This paper will be investigating these potentials and expands the idea on proposing a sustainable architecture as well as a context aware environment.

\section{B. Service Enablers}

In tradition, service implementations have been done by integrating different components vertically and per service. Re-utilization of components has not been considered due to the lack of standards. This silo approach impedes the rapid deployment for new services and raises costs, common functions are duplicated among services, etc.

The new approach using concepts of service enablers provides service environment with interoperable components leveraging the interaction between components and applications developed by different providers. This approach reduces deployment efforts by means of reusing common function components and allows interoperability across a wide variety of environments in a consistent manner.

In order to support the development of applications from different service providers, OMA [6] has specified the OMA Service Environment (OSE) and the specifications of these so called service enablers. It is a flexible and extensible architecture where service enablers perform their potentials always pursuing the reutilization of components and avoiding the reinvention of wheels. OSE comprises: the enablers, enabler components and the interactions between them.

OMA has already developed the specifications for several service enablers that use IMS functions and reference points, i.e. Push to talk Over Cellular (PoC), Presence Simple and SIP Push. On our research project, amongst others, we proposed our own enabler call Location (Fig. 2) for various application cases of location enabled service categories. This paper is about to present our design and prototyping of this enabler and its application on end user services.

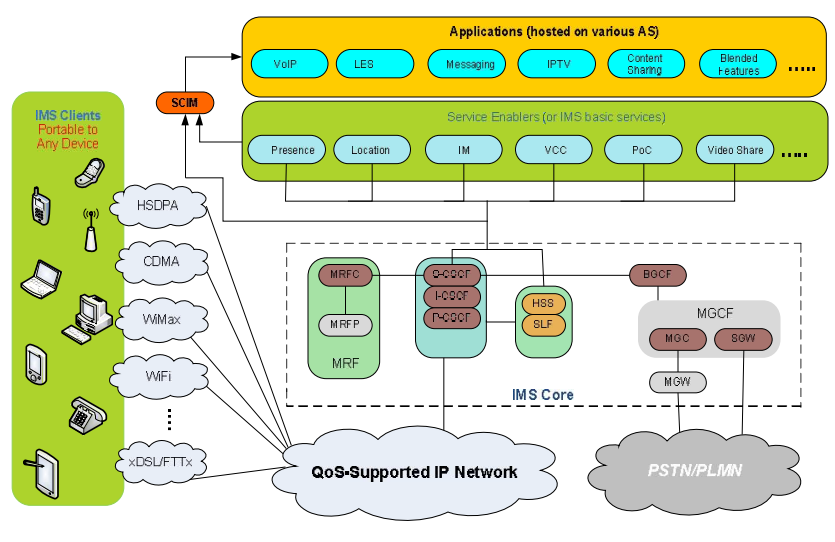

Figure 2. Service Enablers on IMS Framework

\section{SYSTEM DESIGN}

Our concept of location enabled services is a bit different from the tradition. Instead of offering separate applications providing specific stand-alone LBS services, location is increasingly positioned as an enabler of existing mobile services. The transparent use of location results in increased interest in privacy protection, new business/monetization models, web/widget and open source development environments, changing value chain, alternative positioning technologies, new content sourcing paradigms, and innovative interfaces.

But the biggest difference of our work here is that we propose a comprehensive framework (figure 3 ) for providing the (location enabled) service, charging and control it via policy enforcement entities. This is advanced design (compare to OMA location enabler concept [5]) because it helps to make LES to be realized and deployed in commercial environment.

Finally our design provides users with various intelligent communication and social-interaction features based on their current location. Some of them are:

Tracking the (IMS) users: This feature allows someone to track the other users (friends, colleges) via location function of terminal GPS (embedded on 


\section{Research, Development and Application on Information and Communications Technology}

most of smart-phones currently) and/or BTS/NodeB location.

The location sharing: allows someone to share its location to other users and in revert they also can have the current location of others.

Finding the specific facilities (ATM machine, restaurant, etc): allows user to find the near-by posts like the-restaurants, petro stations, etc. on her/his smart-phone map and equipped with the navigation and communication features too.

Management of location data: allows users to grant rights to who can access and/or retrieve this data.

\section{A. Overview of the proposed framework}

Figure 3 presents our overall proposed design for a unified system of Software Defined Network [23] based Multimedia Services in IMS networks. The proposed design contains four subsystems, the first one is IMS Core which are the SIP servers that takes care of routing SIP messages as well as the enforcing (PCRF) control/charging policies on the network infrastructure (open-flow [23] switches); the second one is a sophisticated software framework for end user devices thus provides users with various functions from multimedia communication applications like voice, messaging, IPTV, LES to advanced features like scoring the application quality \& experience. The third subsystem is about the content provider which includes of different ASes for different applications (video for IPTV, LES for location based services, etc), an SCIM module and various service enablers for providing service blending/composition functionalities and more importantly a database of the collected parameters of QoS and QoE Scores from the network infrastructure as well as the users. The last one is an OSS (Operation \& Support Subsystem) that in our design will provide the policy \& charging control mechanisms. As depicted on the figure 3, this subsystem contains different functional blocks for different applications like GARC (Generic Adaptive Resource Control) for containing logics of policy control mechanisms, OF Control for communicating to open-flow switches, CDF and OCS for charging functions. Besides those four subsystems, we also proposed a lot of protocols for linking different functional blocks together; some of them are the standard 3GPP protocols while some are our newly proposed or customized one.

This is, in fact, our national project which divided into few sub-projects with its own results. In this paper, we will only focus on our design and implementation of functionalities and interfaces related to the Location Enabled Services (LES). Therefore, we come to see more specific architecture of LES on Figure 4. Regarding to this LES design, we adopted a standard-based strategy in which the services will be invoked on a standard platform (Parlay-X AS) using our extended interfaces with static and dynamic rules. These (customized and extended) rules are defined and stored on HSS database as usual. This approach makes no change to standardized architecture of IMS (neither adding new functional blocks nor creating totally new interfaces). What new here is that we propose new invocation mechanisms and rules which operated in state-full SIP proxy server and modify HSS database and Sh interface to carry more functions and information that allows the combination of user services as well as service capabilities. The architecture combines of IMS Core (CSCFs), Application Server (LES), User \& Services Database (HSS) and Charging Components (OCS, CDF \& BS) and again, when do the design we try the best to follow most of $3 \mathrm{GPP}$ and TISPAN standards.

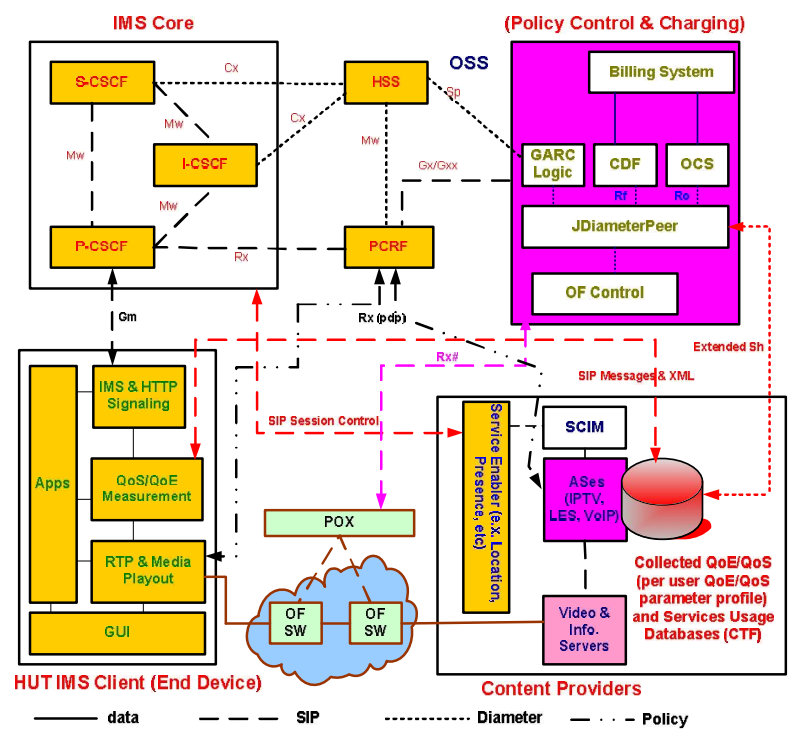

Figure 3. Functional design of the proposed framework 


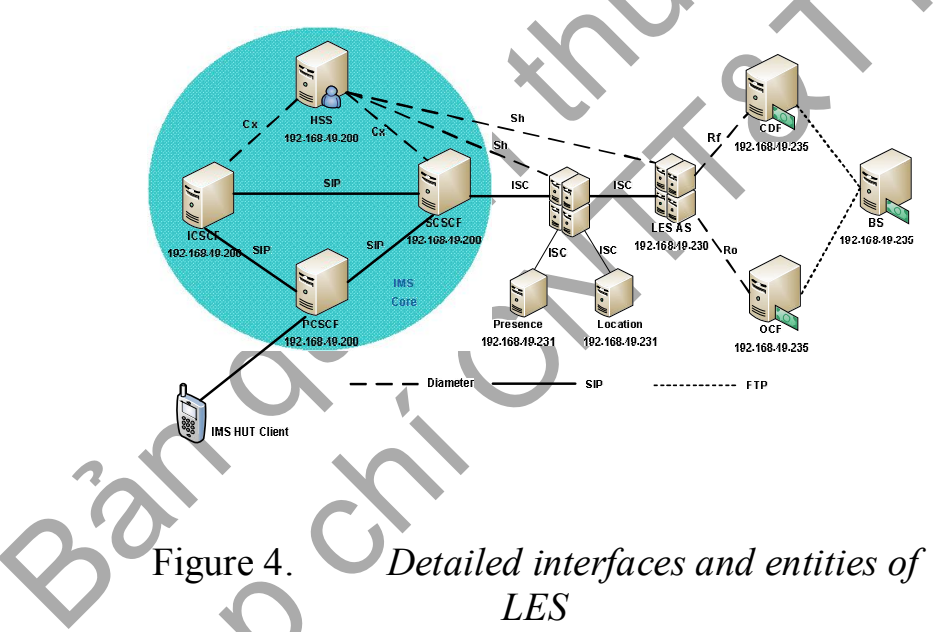

Architecturally, we are the first one that proposes a comprehensive framework which combines all of aspects of a multimedia service, namely, service composition, service control and charging, \& service quality evaluation. Following parts will explain the details of our designs and how it operated.

\section{B. Service Requirements (of LES)}

The proposed architecture meets following advanced requirements on providing the locationenabled services:

Service combination model: on this model, service processes are categorized into two types, basic services or service capabilities (in OMA language are Service Enablers) and type 2 are user services. The service capabilities will have to register itself at the SCIM entity of the framework so that user service processes can automatically discovery it.

Service Invocation: we extended the $\mathrm{iFC}$ on the HSS to add in more information and rules so that SCIM can, thorough extended Sh interface, download it for a flexible and context-ware invocation of a specific service process whenever it need to provide end user with a blending service. The invocation mechanism on this framework allows service processes, to be invoked, hosting on both native SIP AS and Web 2.0 XML service control platforms.

Service Serialization: the framework also supports the function that allows invoking different services on serial order by static rules.

Routing and balancing load between ASes: there may be a scenario that on a network there are multiple
ASes hosting the same services and in that case SCIM will have knowledge of that (through downloading iFC from HSS) in order to allow balancing the load between the ASes.

Finally, User Indentifying: the framework can provide the invoked service process with the user identity (for purpose of charging, for example).

\section{Service Operation}

A series of figures below show the design of procedures and messages for invocation and delivery of the location enabled services to end users. First of all, figure 5 depicts the registration procedure in which, as usual, before using any (location enabled) services the end user/device need to register on the network. This registration procedure is totally standard one of $3 \mathrm{GPP}$.

After registration, the next (Fig. 6) is the location update procedure on which end device (with GPS enabled or CID) automatically and periodically send its Geo position to the LES AS thus allow AS always has up-to-minute position data of all the clients. Before updating its location, client has to setup a session to S-CSCF and then to LES AS with an INVITE message. After that it send its location via message PUBLISH of SIP Event package which contains location information on XML format as below:

$$
\begin{aligned}
& <? x m l \text { version }=" 1.0 " \text { encoding }=" U T F-8 " ?> \\
& <\text { lbs name }=\text { "lbs.ims.hut.vn"> } \\
& <\text { tuple uri="sip:alice@ims.hut.vn"> } \\
& <\text { longitude type }=\text { "int"> } \\
& <\text { longitude }> \\
& <\text { latitude type }=" \text { int" }> \\
& 21003953 \\
& <\text { latidude }> \\
& <\text { accuracy type }=\text { "int" }> \\
& 39 \\
& </ \text { accuracy }> \\
& </ \text { tuple }> \\
& </ l b s>
\end{aligned}
$$

Figure 7 shows the location tracking procedure on which a user has capability to monitor the location of other user/device, of course given the acceptance of tracked users and that the tracked user has frequently update its location into LBS AS as on figure 5. Similarly, the tracking procedure, first also the client has to setup a session to SCSCF and LBS AS as updating procedure. That also means the client 
Research, Development and Application on Information and Communications Technology

already register to the SIP Event package, this every time, other users updating their position, the registered user will receive that location information via NOTIFY message on XML format again.
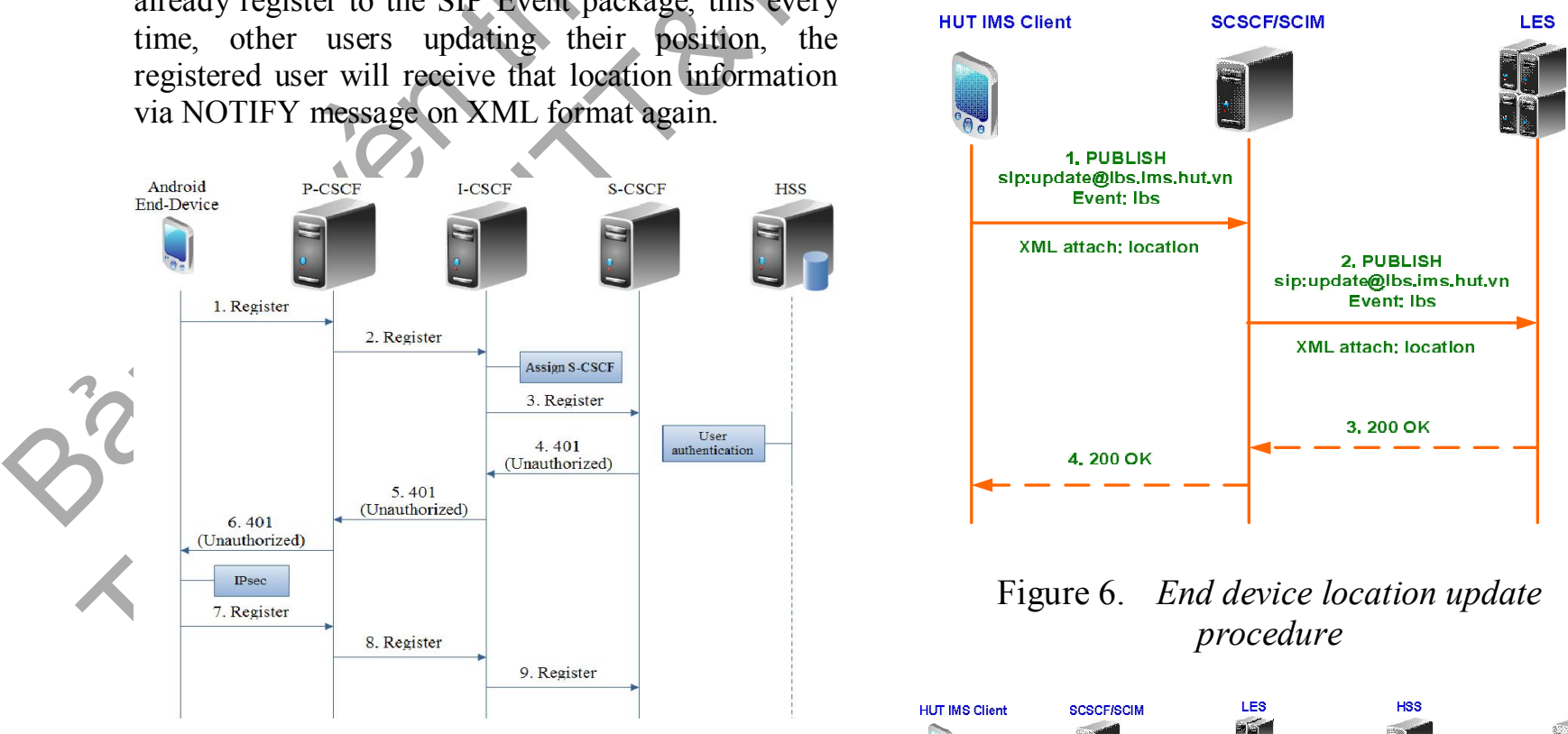

Figure 5. Registration Procedure

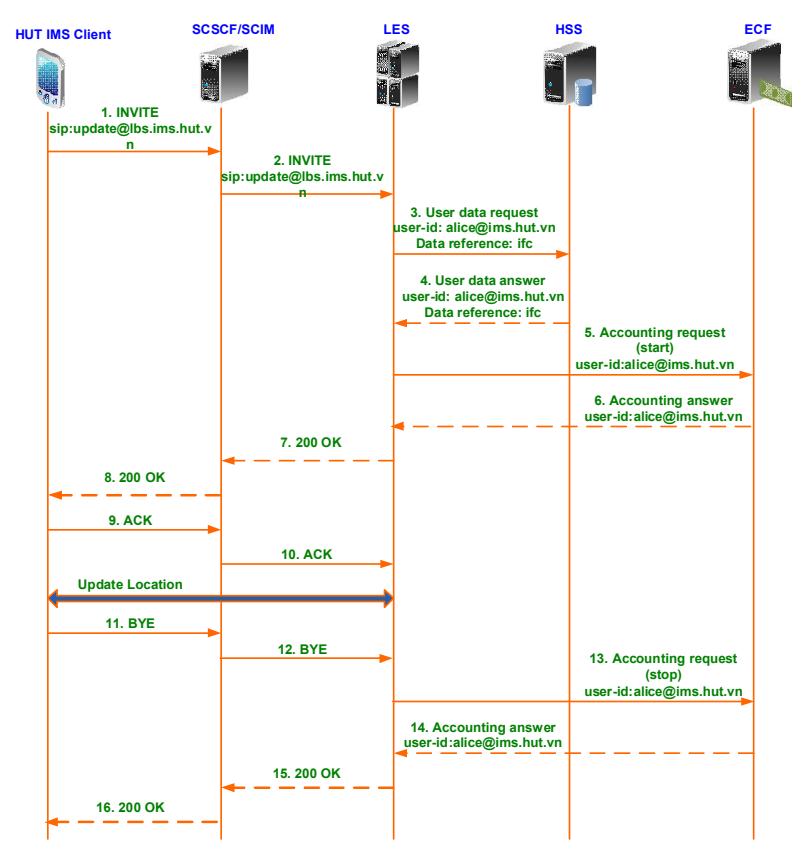

Figure 6. End device location update procedure

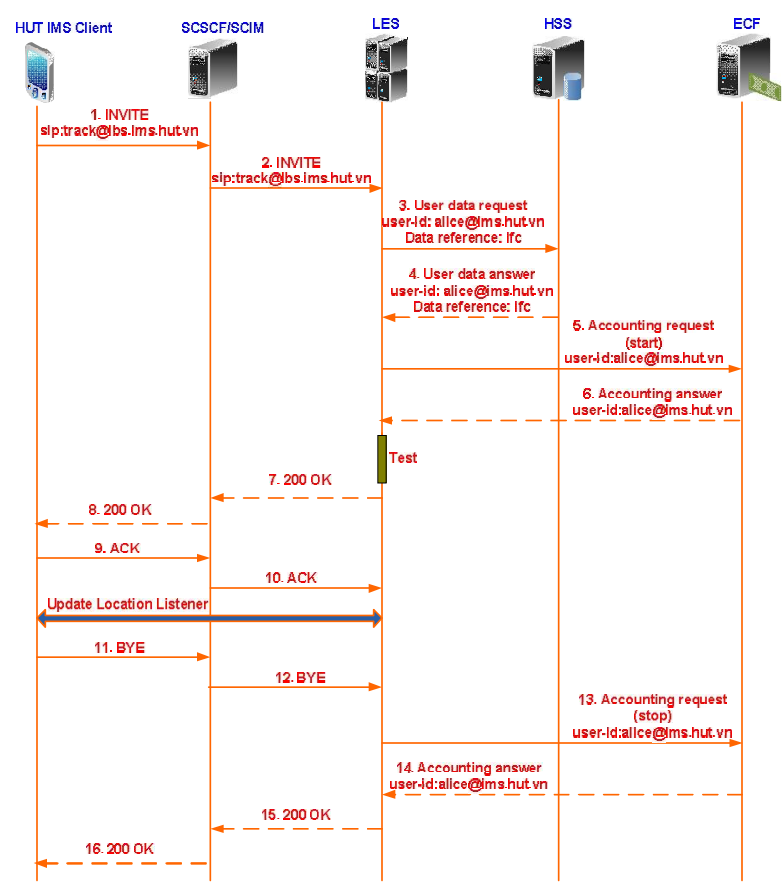




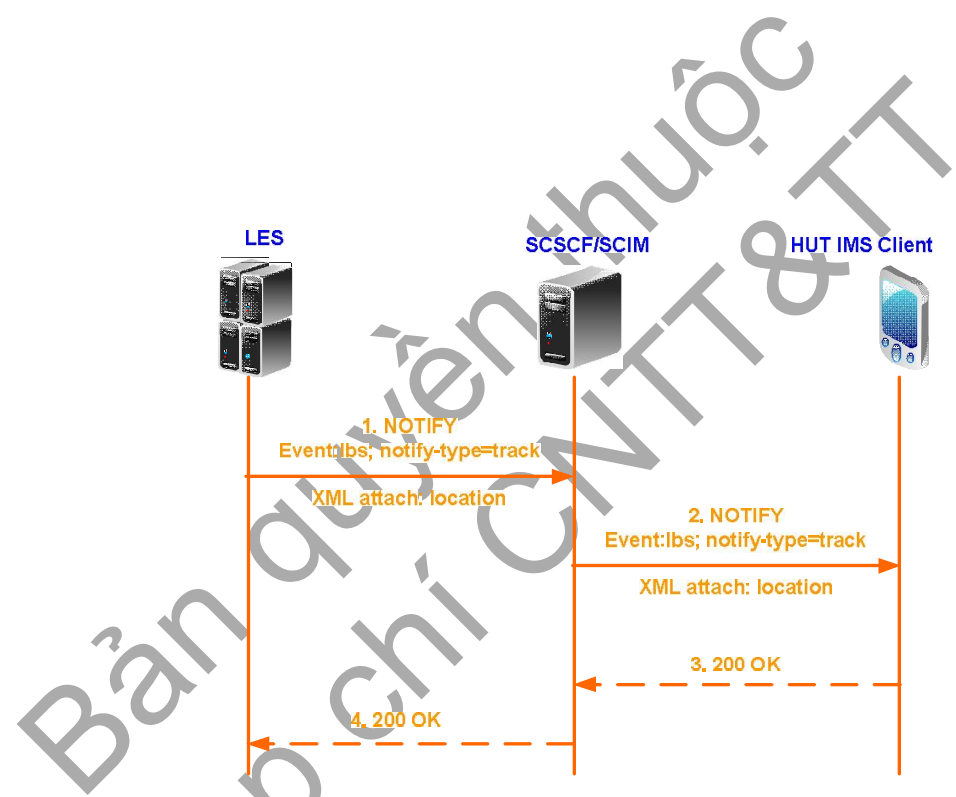

Figure 7. Location tracking procedure

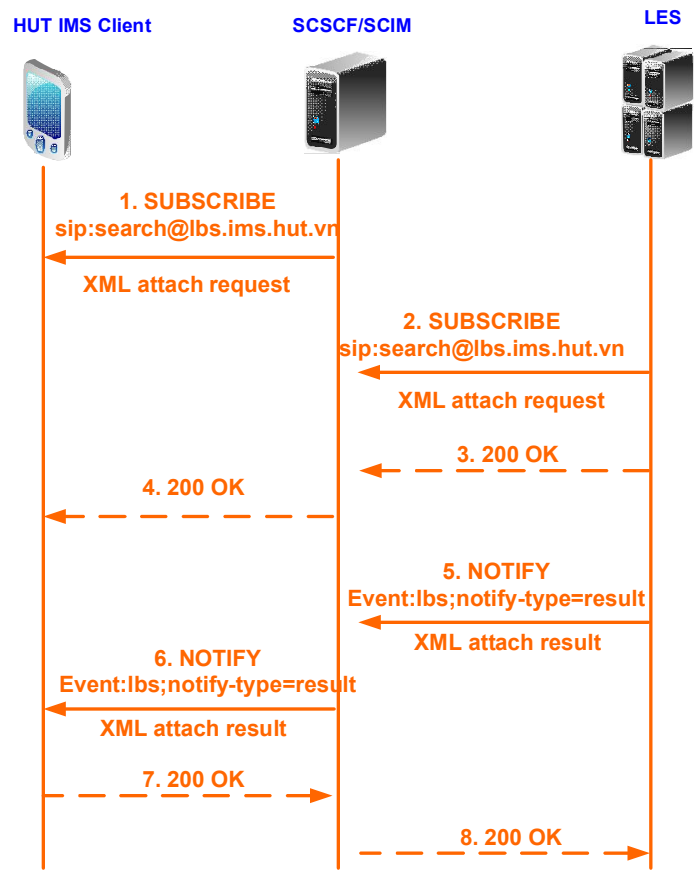

Figure 8. Location finding procedure

Figure 8 depicts the location finding procedure on which allows any IMS user to find the specific location (restaurant, public locations) on the LBS database. This procedure's also using the SUBCRIBE and NOTIFY message of the SIP EVENT Package for purpose of querying and retrieving the location information on XML format again. Finally, though not depicted on these figure, but our design also allows administration procedure of those location information to ensure the privacy of the collected info. Specifically, it allows user to specify, via real time signaling, who has right to access its location information as well as who can track it location.

\section{SYSTEM IMPLEMENTATION}

From the above conceptual design, we implement the proposed architecture end to end, from the Client on the Android platform to LES AS, extended HSS database to the Policy/Charging Control (of location enabled services) components. This section presents major parts of such implementation.

\section{A. IMS HUT Client}

As stated above, our sophisticated IMS Client Software on Android platform, not only provide location enabled services but also for various other applications like IPTV, VOIP, Messaging, and Composited Intelligent Service Features. Figure 9 below shows the functional diagram of the software. Regarding to the LES, several functional blocks have been implemented for various procedures of registration, location updating, location management, location tracking and location finding. And figure 10 depicts the UML diagram of the location tracking procedure as an example. Finally, Fig. 10 shows the software classes of the LES design.

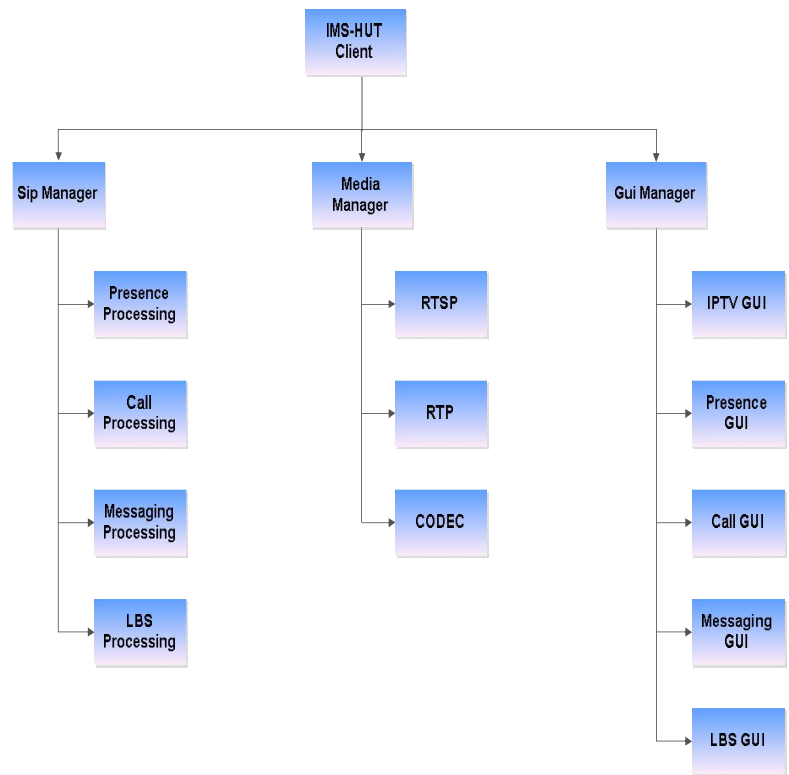

Figure 9. Functional diagram of IMS HUT Client 


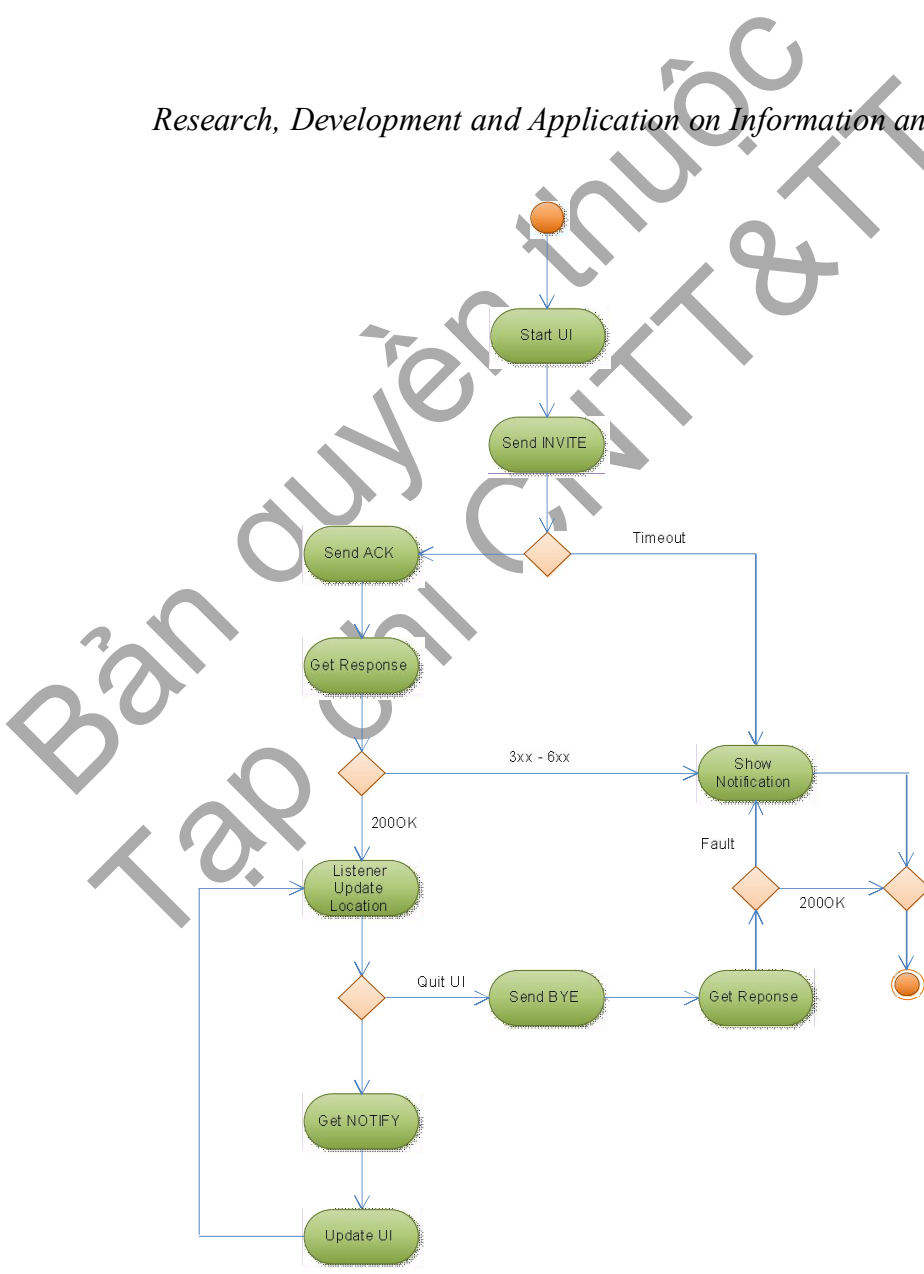

Figure 10. UML diagram of location tracking procedure on IMS HUT Client

\section{B. LES Application Server}

On the service provider side, we implement the AS platform using the SIP Servlet [21] and Parlay-X [22] technologies. The servers have been designed and implemented with major functionalities of (1) hosting the service logics on Parlay X API; (2) storage of user and resources information; (3) finding the resources based on location and or searching key words; (4) tracking the locations; (5) periodically collected QoE Scores from users and QoS parameters from network nodes; and (6) interfacing with HSS module and Presence Server to load the user profiles and status.

Like the client side, ASes were implemented with several procedures: updating user location to LES AS database, setting up the tracking list, tracking user location and its status and finding the location from database. Figure 12 shows the UML diagram for user tracking procedure as an example, while 13 depicts its software class design and figure 14 is the algorithm to find a specific location (ATM) on LES AS.

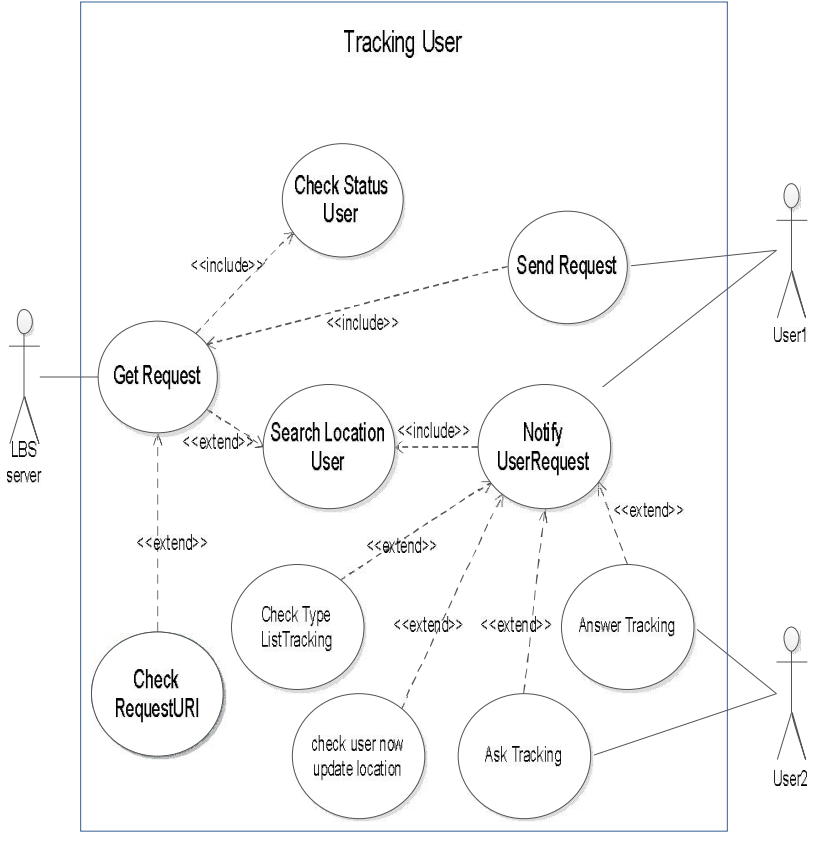

Figure 11. Sh-interface extended data

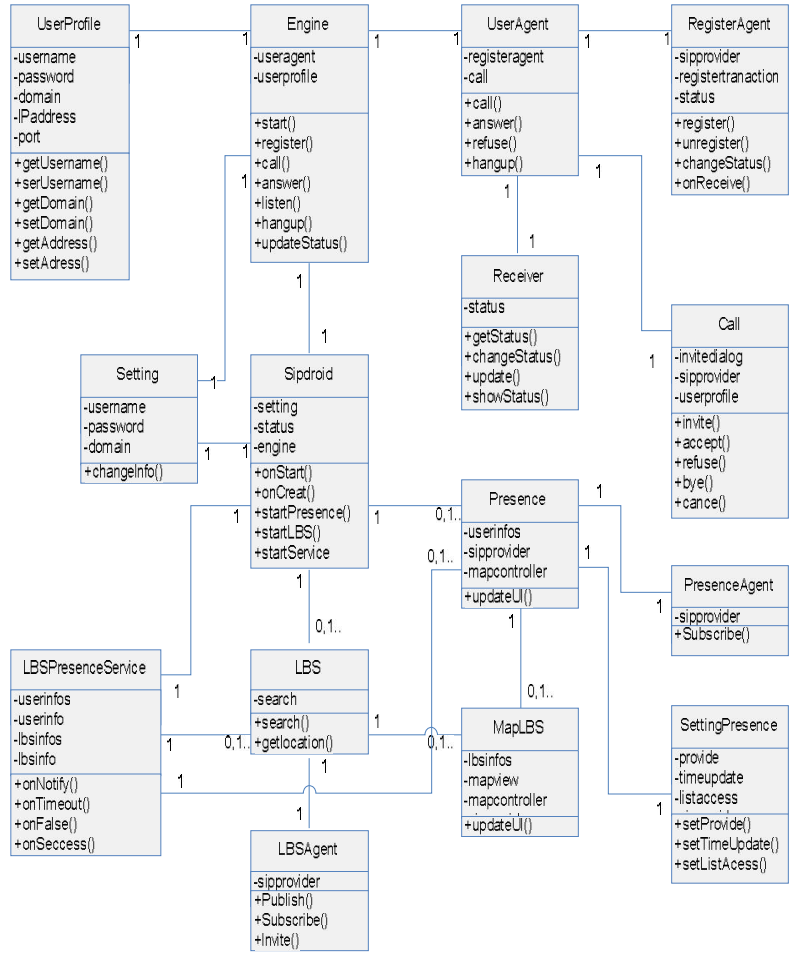

Figure 12. UML diagram for user tracking on $L B S A S$ 
P. Plicy Control \& Charging

\section{Policy Control \& Charging Modules}

As stated on the section IV-A above, one of our contribution on this work is linking up between the main (location enabled) service mechanisms with necessary charging and policy control functions in an overall architecture, thus, besides the software on the client and server sides, we implemented several software modules for policy enforcement and charging tasks. However, in this paper we just want to highlight the innovative ideas of this linkage. That said, our proposed framework (Figure 3) for this purpose was done with two protocol stacks, OF Control is for communicating with Open-flow controller $(\mathrm{POX})$, the other is JDiameterPeer for communicating with application platform. On top of the Diameter stack are three main functional services, the GARC hosting the logics of different policies for enforcing QoS mechanisms on the OF switches/routers based on collected user's perception of service quality (QoE values). The OCS and CDF are two blocks implementing the charging function for services providing to end users. The charging mechanisms provided on our implementation are very flexible, it could be based on session duration; quality of service; exchanged data volume or based on events happened.

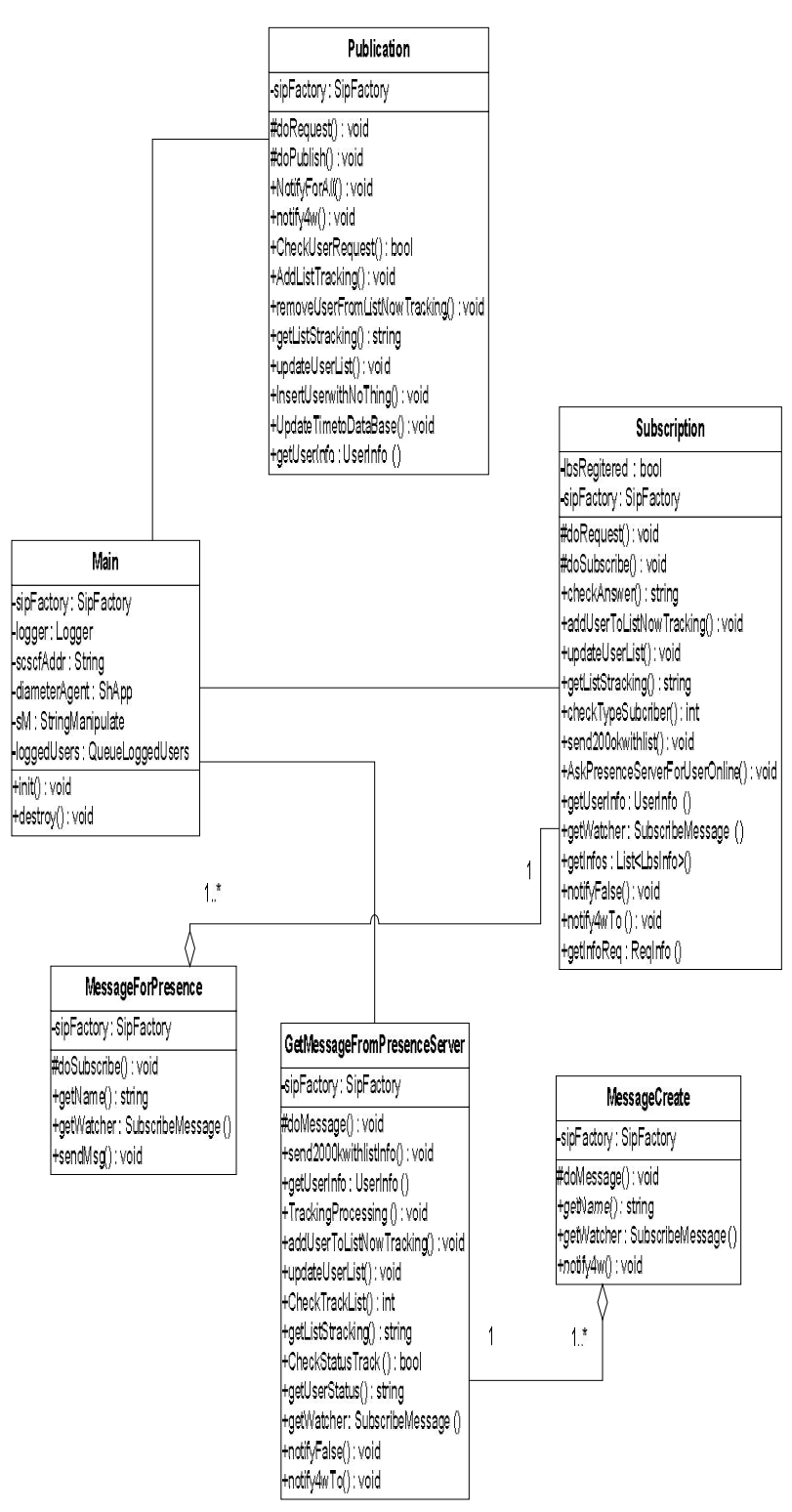

Figure 13. Class design of user tracking procedure on $L B S A S$ 


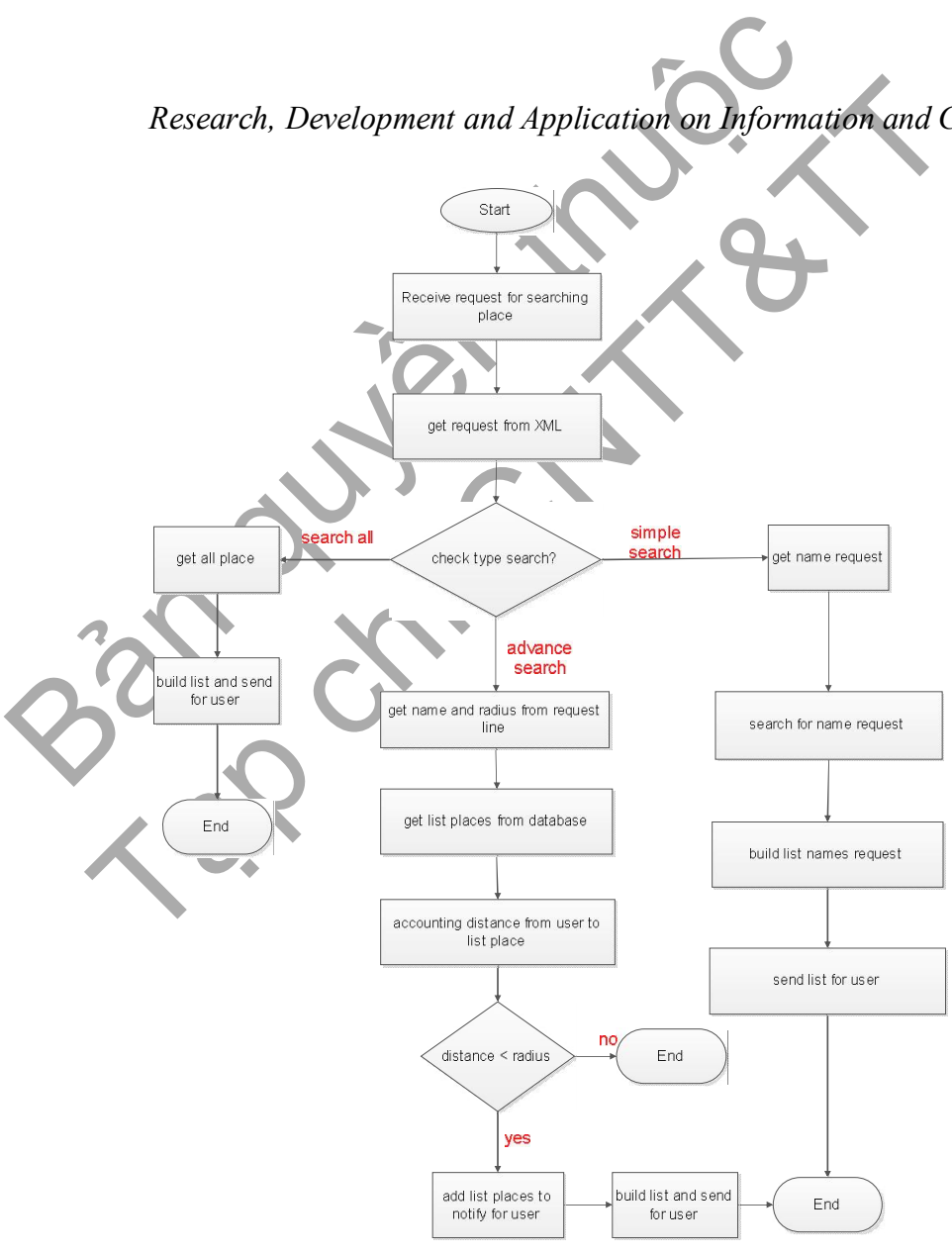

Figure 14. Algorithm for finding a specific place

\section{TESTBED AND PROTYPING}

In scope of our national research project as mentioned above, "Development of the new service framework for $3 \mathrm{G} / 4 \mathrm{G}$ and broadband Internet using IMS technology", we had designed and built a unified $\&$ federated test-bed platform using most of open sources and in-house developed components and some commercial hardware. As depicted on figure 15, the test-bed spans from client software for both mobile \& fix users, to the IMS core networks, Application platforms, Information center, content provider and policy \& charging control.

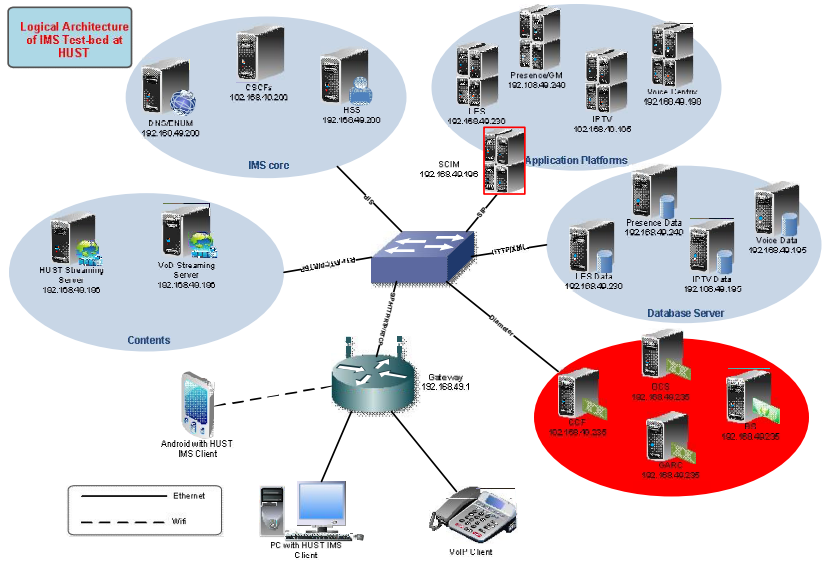

Figure 15. HUT IMS Test-bed

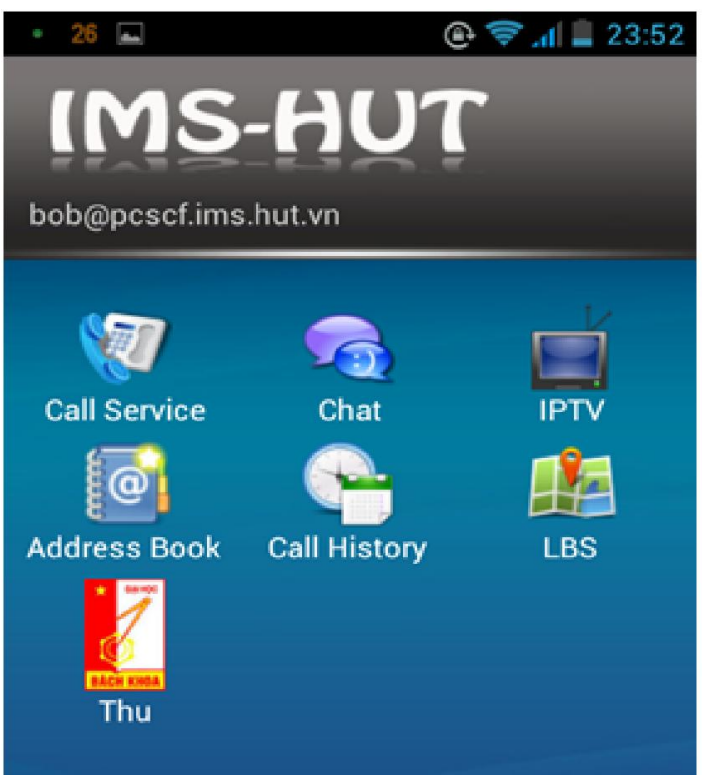

Figure 16. GUI of HUT IMS Client on Android with various categories of multimedia and location enabled servcies and control functions

Figure 16 shows the GUI of our own developed HUT IMS Client for smartphone that runs Android OS. The Client allows the users to use multiple multimedia services from the basic one like broadcast TV, contents on demand, VOIP, messaging, online address book to intelligent one like incoming call/message pop-up (on movies session) to location enabled services. Figure 17 shows an example of advanced feature of LES that automatically pop-up the near-by public places (with the contact details like telephone no. and web address which can initiate 
communication sessions online) and/or friends (with their current status) when user enter a place.

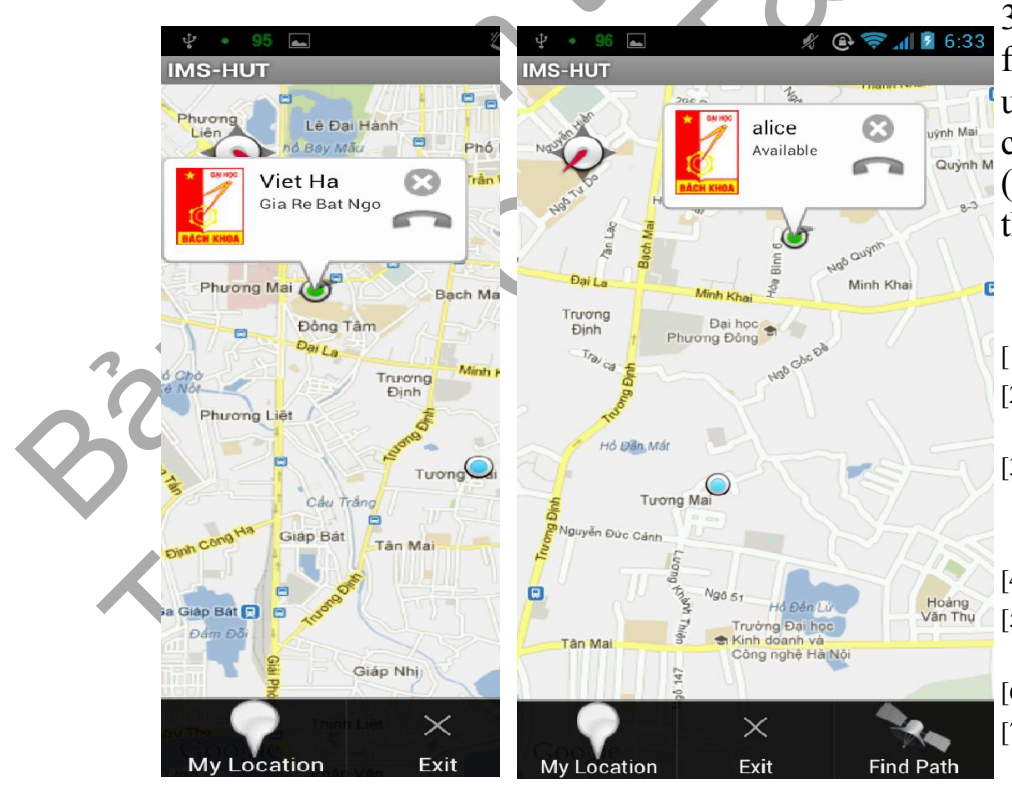

Figure 17. The results of finding a specific place (Viet Ha shop) and another user (Alice)

These prototyping results are proof-of-concept for our newly proposed architecture on Fig. 3 which makes linkage of the service framework (IMS) with the underlying network infrastructure (OpenFlow) and pave the way for a new research direction of providing multimedia services with capabilities of quality (QoS \& QoE) assurance and policy enforcement which are very important in this modern communication world. Finally, our federated test-bed platform provide a comprehensive enviroment for conducting new research projects in the field of multimedia communication as well as the service deployment trials of industrial partners.

\section{CONCLUSION AND FUTURE WORK}

This paper presents the details of the location enabled service design and implementation as a proofof-concept for our newly proposed multimedia communication frame-work which contains all of needed functionalities from service providing to service composition to quality assurance and policy control. Our research had pointed out the necessary of designating the basic location related functions into so-called location enabler and linkage of it to policy and charging control functions in order to make it be consistent with service concept of modern world nowadays. These results pave firm initial steps on the way toward completing the service platform of the 3GPP's IMS architecture in NGNs. The research is in fact our on-going works; we currently work on the user cases (of LES) that make combination of service capabilities from SIP platform and pure Web (UDDI/SOAP) platform and enhancing \& completing the policy control and charging functions.

[1] TS 23.328, "IP Multimedia Subsystem," 3GPP, Release 6

[2] J. Rosenberg, et al., "SIP: Session Initiation Protocol," RFC 3261, IETF, June 2002

[3] F. inc., XDMS: The FOKUS XML Document Management Server,

http://www.fokus.fraunhofer.de/bereichsseiten/.

[4] Open IMS Core Playfround, see http://www.openimscore.org/

[5] http://technical.openmobilealliance.org/Technical/released enab lers.aspx

[6] http://openmobilealliance.org/

[7] M. Handley, V. Jacobson, "SDP: Session Description Protocol," RFC 2327, IETF, April 1998

[8] www.3gpp.org

[9] Jonathan Rosenberg. RFC 4825: The Extensible Markup Languag (XML) Configuration Access Protocol (XCAP). Internet Engineering Task Force, May 2007.

[10] T. Koskela et al, "Towards Context-Aware Mobile Web 2.0 Service Architecture", Proc. UBICOMM'07., Papaete, 2007

[11] N.Banerjee, K. Dasgupta and S. Mukherjea, "Providing Middleware Support for the Control and Co-ordination of Telecom Mashups", Proc. USENIX Middleware 2007, Newport, 2007

[12] B.Falchuk, S.Loeb, T.Panagos, "A Deep-Context Personal Navigation System", Proc. ITS America 15th World Congress on Intelligent Transportation Systems, New York, 2008

[13] M. Poikselka, G. Mayer, H. Khartabil, A. Niemi, The IMS: IP Multimedia Concepts and Services. 2nd ed. West Sussex, England: John Wiley and Sons Ltd, 2006

[14] 3GPP TR 23.810 V8.0.0 (2008-09), Study on Architecture Impacts of Service Brokering (Release 8)

[15] Goveas, R.; Sunku, R.; and Das, D.; "Centralized Service Capability Interaction Manager (SCIM) architecture to support dynamic-blended services in IMS network", presented at 2nd International Conference on Internet Multimedia Services Architecture and Applications IMSAA 2008, pp 1-5, December 2008, doi: 10.1109/IMSAA.2008.4753933

[16] Gouya, A.; Crespi, N.; Bertin, E.; , "SCIM (Service Capability Interaction Manager) Implementation Issues in IMS Service Architecture," Communications, 
Research, Development and Application on Information and Communications Technology

2006. ICC '06. IEEE International Conference on , vol.4, pp.1748-1753, June 2006, IEEE

[17] J. M. Polk, B. Rosen, "Requirements for Session Initiation Protocol Location Conveyance", IETF Internet-Draft, draft-ietf-sipping-locationrequirements-02.txt, October 25th, 2004

[18] Elmangosh, A.; Ashibani, M.; Ventura, N,; BenShatwan, F; "Service Brokering in IP Multimedia Subsystem,', in Proceedings SATNAC, 2009

[19] Blum, N.; Magedanz, T.; Schreiner, F.; , "Management of SOA based NGN service exposure, service discovery and service composition," Integrated Network Management, 2009. IM '09. IFIP/IEEE International Symposium on, pp.430-437, 1-5 June 2009, IEEE Press Piscataway, NJ, USA

[20] Brajdic, A.; Lapcevic, O.; Matijasevic, M.; Mosmondor, M.; , "Service composition in IMS: A location based service example," Wireless Pervasive Computing, 2008. ISWPC 2008. 3rd International Symposium on , vol., no., pp.208-212, 7-9 May 2008, doi: 10.1109/ISWPC.2008.4556198.

[21] http://jcp.org/aboutJava/communityprocess/final/jsr28 9/

[22] www.parlayx.com

[23] OpenFlow Project and Protocol, http://www.openflow.org/

\section{AUTHOR'S BIOGRAPHY}

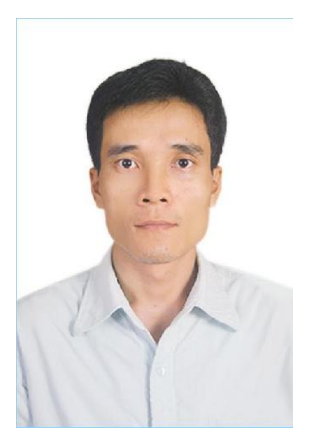

Nguyen Tai Hung (PhD) is lecturer in the faculty of electronic $\&$ telecommunication at the Hanoi University of Science \& Technology in Hanoi, Vietnam. He got his Master and $\mathrm{PhD}$ degrees, both in communication engineering, from same university in 2001 and 2007, respectively. He is the (co)author of around thirty of scientific papers/articles and a national research project in the fields of Internet engineering and next generation networks on international journals and conference proceeding. He spent a half of year in 2008 with Fraunhofer Institute of Fokus in Berlin, Germany for conducting the research project of service development for 3G/NGN networks. Based on his more than 10 years of experience in the teaching complex IT and telecommunication technologies to different courses in an easy to digest way, Dr. Nguyen Tai Hung gains an extensive knowledge in field of the next generation networks and the Future Internet. 